\title{
From a domain analysis to the specification and detection of code and design smells
}

\author{
Naouel Moha ${ }^{1,2,4}$, Yann-Gaël Guéhéneuc ${ }^{3}$, Anne-Françoise Le Meur ${ }^{2}$, \\ Laurence Duchien ${ }^{2}$ and Alban Tiberghien ${ }^{2}$ \\ ${ }^{1}$ Ptidej Team, GEODES, DIRO, Université de Montréal, Montreal, QC, Canada \\ 2 Adam Team, INRIA Lille Nord Europe, LIFL CNRS UMR 8022, Université des Sciences et Technologies de Lille, \\ Villeneuve-d' Ascq cedex, France \\ ${ }^{3}$ Ptidej Team, DGIGL, École Polytechnique de Montréal, Montreal, QC, Canada \\ ${ }^{4}$ IRISA, Triskell Team, INRIA Rennes-Bretagne Atlantique, Campus de Beaulieu, 35042 Rennes cedex, France. E-mail: moha@irisa.fr
}

\begin{abstract}
Code and design smells are recurring design problems in software systems that must be identified to avoid their possible negative consequences on development and maintenance. Consequently, several smell detection approaches and tools have been proposed in the literature. However, so far, they allow the detection of predefined smells but the detection of new smells or smells adapted to the context of the analysed systems is possible only by implementing new detection algorithms manually. Moreover, previous approaches do not explain the transition from specifications of smells to their detection. Finally, the validation of the existing approaches and tools has been limited on few proprietary systems and on a reduced number of smells. In this paper, we introduce an approach to automate the generation of detection algorithms from specifications written using a domain-specific language. This language is defined from a thorough domain analysis. It allows the specification of smells using high-level domain-related abstractions. It allows the adaptation of the specifications of smells to the context of the analysed systems. We specify 10 smells, generate automatically their detection algorithms using templates, and validate the algorithms in terms of precision and recall on XERCES v2.7.0 and GANTTPROJECT v1.10.2, two open-source object-oriented systems. We also compare the detection results with those of a previous approach, IPLASMA.
\end{abstract}

Keywords: Design smells, Antipatterns, Code smells, Domain-specific language, Algorithm generation, Detection, JAVA.

\section{Introduction}

Following their development and delivery to the clients, software systems enter the maintenance phase. During this phase, systems are modified and adapted to support new user requirements and changing environments, which can lead to the introduction of "bad smells" in the design and code. Indeed, during the maintenance of systems, their structure may deteriorate because the maintainer's efforts are focused on bugs under cost and time pressure, thus with little time to keep the code and design "clean".

Correspondence and offprint requests to: N. Moha, E-mail: moha@irisa.fr 
Yet, code and design smells are costly because these "bad" solutions to common and recurrent problems of design and implementation are a frequent cause of low maintainability ${ }^{1}$ [Pre01] and can impede the evolution of the systems. During formal technical reviews, quality experts may detect errors and smells early, before they are passed on to subsequent software development and maintenance activities or released to customers. However, the detection of code and design smells requires important resources in time and personal, as well as being error-prone [TSFB99].

Code and design smells are problems resulting from bad design practices [TSFB99]. They include problems ranging from high-level and design problems, such as antipatterns [ $\left.\mathrm{BMB}^{+} 98\right]$ (that we will designate as design smells in the rest of the paper), to low-level or local problems, such as code smells [Fow99]. Code smells are in general symptoms of design smells. However, software engineers can define their own code and design smells according to their own understanding and the context of their systems and organisations.

One example of a very common design smell is the Blob [BMB ${ }^{+}$98, p. 73-83], also known as God Class [Rie96]. The Blob reveals a procedural design (and thinking) implemented with an object-oriented programming language. It manifests itself through a large controller class that plays a God-like role in the program by monopolizing the processing, and which is surrounded by a number of smaller data classes providing many attributes but few or no methods. In the Blob, the code smells are the large class and its surrounding data classes.

Several approaches [AS06, Mar04, Mun05] and tools [Flo02, HP04, PMD02] have been proposed in the literature to specify and detect smells. Although showing interesting results, these approaches have the following limitations:

- Problem 1: Lack of high-level specifications of smells adapted to analysed systems. These approaches propose the detection of a set of predefined hard-coded smells. The detection of new smells requires a new implementation using programming languages. The detection algorithms are defined at the code level by developers rather than at the domain level by quality experts. Moreover, the implementation of the detection algorithms is guided by the services of the underlying detection framework rather than by a study of the textual descriptions of the smells. Lincke et al. [LLL08] demonstrate that the problem is even worst, in particular among metrics-based tools. They show that metrics-based tools not only interpret and implement the definitions of metrics differently but also deliver tool-dependent metrics results, and these differences have implications on the analyses based on the metrics results. Consequently, it is difficult for quality experts not familiar with these languages and services to evaluate the choices made by the developers, to adapt the algorithms or specify new detection algorithms to take into account the contexts of the analysed systems.

- Problem 2: "Magical" transition from the specifications of smells to their detection. Most of the approaches do not explain the transition from the specifications of smells to their detection. The way specifications are processed or manipulated is not clear and the definition of the underlying detection platform that allows the detection is not documented. This lack of transparency prevents replication and improvement of detection techniques, but also the technical comparison of the implementation of these techniques.

- Problem 3: Partial validation of the existing detection techniques. The results of the detection with the approaches are not presented on a representative set of smells and on available systems. Indeed, available results concern proprietary systems and a reduced number of smells.

In this paper, we follow the principle of domain analysis [PD90] to propose a domain-specific language to specify code and design smells at the domain level and generate automatically detection algorithms from these specifications. A shorter version of this paper was published in [MGMD08]. We extend this previous version of the paper with (1) a more significant set of smells, (2) detection results on a second open-source system, and (3) an extended description of the domain analysis that gives a new research point of view with respect to our previous work [MGL06, MHG06]. Figure 1 illustrates the following contributions:

- Contribution 1: We describe and implement a domain-specific language, 2D-DSL (Design Defects Domain Specific Langage) based on a unified vocabulary that allows quality experts to specify smells. This language results from a thorough domain analysis of smells, allows quality experts to take into account the contexts of the analysed systems, and allows the generation of traceable detection algorithms.

\footnotetext{
1 Maintainability is defined as "the ease with which a software system or component can be modified to correct faults, improve performance, or other attributes, or adapt to a changed environment" [Ger91].
} 


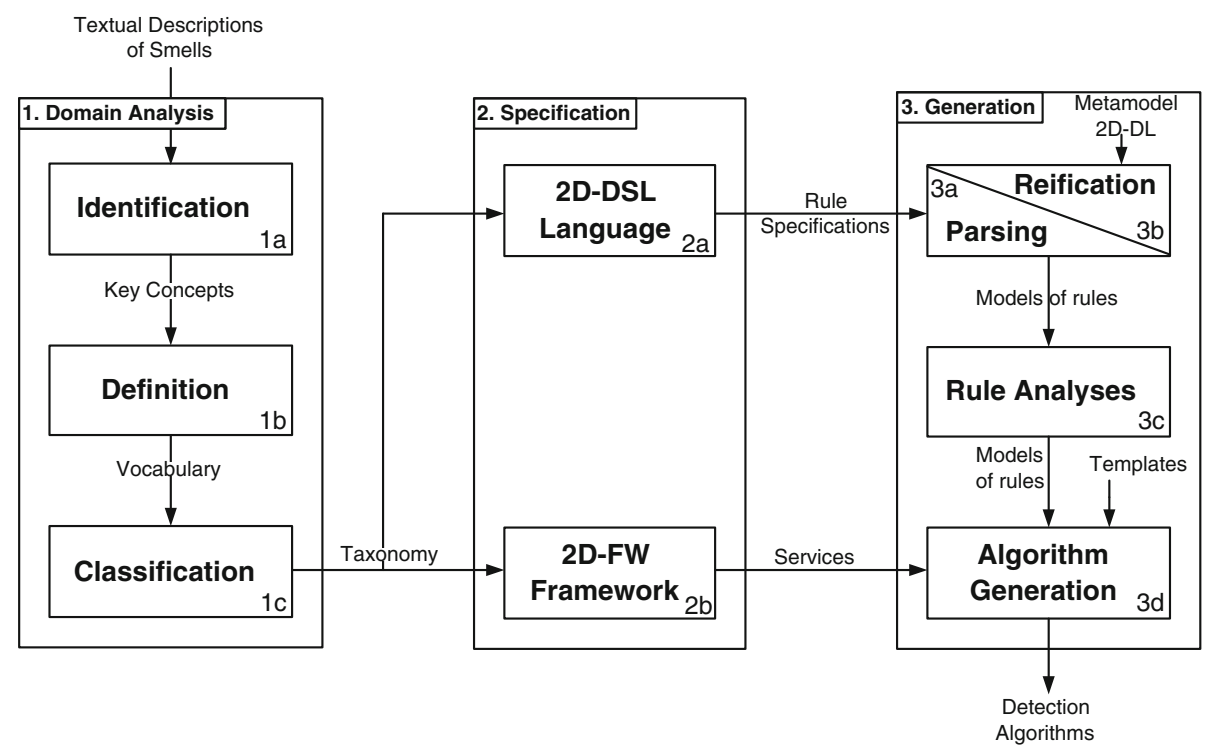

Figure 1. Generation process

- Contribution 2: We propose an explicit process for generating detection algorithms automatically using templates. We recall the services provided by our underlying detection framework, 2D- FW (Design Defects FrameWork), which allow the automatic generation of detection algorithms from the specifications. These algorithms correspond to JAVA code source directly compilable and executable without any manual intervention.

- Contribution 3: We present the validation of our process including the first study of both precision and recall on two large open-source software systems, GANTTPROJECT v1.10.2 and XerCes v2.7.0. This validation constitutes a first result in the literature on both the precision and recall of a detection technique on open-source systems and on a representative set of ten smells.

In the rest of this paper, Sect. 2 presents the domain analysis performed on the literature pertaining to smells. Section 3 presents 2D-DSL and 2D- FW. Section 4 describes the generation process of detection algorithms. Section 5 validates our contributions with the specification and detection of ten smells including the antipatterns: Blob, Functional Decomposition, Spaghetti Code, and Swiss Army Knife, and the code smells: Large Class, Lazy Class, Long Method, Long Parameter List, Refused Parent Bequest, Speculative Generality, on the open-source systems XERCES v2.7.0 and GANTTPROJECT v1.10.2. Section 6 surveys related work and explicitely compares our approach to IPLASMA, the current state-of-the-art tool. Section 7 concludes and presents future work.

\section{Domain analysis of code and design smells}

"Domain analysis is a process by which information used in developing software systems is identified, captured, and organised with the purpose of making it reusable when creating new systems" [PD90].

In the context of smells, information relates to the smells, software systems are detection algorithms, and the information on smells must be reusable when specifying new smells. Thus, we have studied the textual descriptions of code and design smells in the literature to identify, define, and organise the key concepts of the domain, Steps 1a-1c in Fig. 1. 
Table 1. List of the four design smells

\begin{abstract}
The Blob (called also God class [Rie96]) corresponds to a large controller class that depends on data stored in surrounded data classes. A large class declares many fields and methods with a low cohesion. A controller class monopolises most of the processing done by a system, takes most of the decisions, and closely directs the processing of other classes [WBM02]. Controller classes can be identified using suspicious names such as Process, Control, Manage, System, and so on. A data class contains only data and performs no processing on these data. It is composed of highly cohesive fields and accessors
\end{abstract}

The Functional Decomposition antipattern may occur if experienced procedural developers with little knowledge of object-orientation implement an object-oriented system. Brown describes this antipattern as "a "main' routine that calls numerous subroutines". The Functional Decomposition design smell consists of a main class, i.e., a class with a procedural name, such as Compute or Display, in which inheritance and polymorphism are scarcely used, that is associated with small classes , which declare many private fields and implement only a few methods.

The Spaghetti Code is an antipattern that is characteristic of procedural thinking in object-oriented programming. Spaghetti Code is revealed by classes with no structure, declaring long methods with no parameters, and utilising global variables for processing. Names of classes and methods may suggest procedural programming. Spaghetti Code does not exploit and prevents the use of object-orientation mechanisms, polymorphism and inheritance .

The Swiss Army Knife refers to a tool fulfilling a wide range of needs. The Swiss Army Knife design smell is a complex class that offers a high number of services, for example, a complex class implementing a high number of interfaces. A Swiss Army Knife is different from a Blob, because it exposes a high complexity to address all foreseeable needs of a part of a system, whereas the Blob is a singleton monopolising all processing and data of a system. Thus, several Swiss Army Knives may exist in a system, for example utility classes

Table 2. List of the six code smells

The Large Class is a class with too many responsibilities. This kind of class declares a high number of usually unrelated methods and attributes

The Lazy Class is a class that does not do enough. The few methods declared by this class have a low complexity

The Long Method is a method with a high number of lines of code. A lot of variables and parameters are used. Generally, this kind of method does more than its name suggests it

The Long Parameter List corresponds to a method with high number of parameters. This smell occurs when the method has more than four parameters

The Refused Parent Bequest appears when a subclass does not use attributes and/or methods public and/or protected inherited by a parent. Typically, this means that the class hierarchy is wrong or badly organized

The Speculative Generality is an abstract class without child classes. It was added in the system for future uses and this entity pollutes the system unnecessarily

\title{
2.1. Identification of the key concepts
}

The first step of the domain analysis consists of reviewing the literature on smells for example the books and articles cited in the related work in Sect. 6, to identify essential key concepts. This step is performed manually so its description would be necessarily narrative. Therefore, to illustrate this step, we use the example of the Spaghetti Code antipattern. We summarise the textual description of the Spaghetti Code $\left[\mathrm{BMB}^{+} 98\right.$, page 119] in Table 1 along with these of the Blob $\left[\mathrm{BMB}^{+} 98\right.$, page 73], Functional Decomposition $\left[\mathrm{BMB}^{+} 98\right.$, page 97], and Swiss Army Knife $\left[\mathrm{BMB}^{+} 98\right.$, page 197] that will be used in the rest of the paper. Table 2 gives the descriptions of the code smells participating in each of these antipatterns.

In the textual description of the Spaghetti Code, we identify the key concepts (highlighted) of classes with long methods, procedural names and with methods with no parameter, of classes defining global variables, and of classes not using inheritance and polymorphism.

We perform this first step iteratively: for each description of a smell, we extract all key concepts, compare them with existing concepts, and add them to the set of key concepts avoiding synonyms, a same concept with two different names, and homonyms, two different concepts with the same name. We study 29 smells, which included 8 antipatterns and 21 code smells. These 29 smells are representative of the whole set of smells described in the literature and include about 60 key concepts. 


\subsection{Definition of the key concepts}

The key concepts include metric-based heuristics as well as structural and lexical information. In the second step, we define the key concepts precisely and form a unified vocabulary of reusable concepts to describe smells. We do not present the definitions of each key concept because it would be very descriptive but we introduce a classification of the key concepts according to the types of properties on which they apply: measurable, lexical, and structural properties.

Measurable properties pertain to concepts expressed with measures of internal attributes of the constituents of systems (classes, interfaces, methods, fields, relationships, and so on). A measurable property defines a numerical or an ordinal value for a specific metric. Ordinal values are defined with a 5-point Likert scale: very high, high, medium, low, very low. Numerical values are used to define thresholds whereas ordinal values are used to define values relative to all the classes of a system under analysis. In the textual descriptions of the Blob, Functional Decomposition, and Swiss Army Knife, the keywords such as high, low, few, and many are used and refer to measurable properties.

These properties also related to a set of metrics identified during the domain analysis, including Chidamber and Kemerer metric suite [CK94]: depth of inheritance DIT, lines of code in a class CLASS_LOC, lines of code in a method METHOD_LOC, number of attributes declared in a class NAD, number of methods declared in a class NMD, lack of cohesion in methods LCOM, number of accessors NACC, number of private fields NPRIVFIELD, number of interfaces NINTERF, number of methods with no parameters NMNOPARAM.

Lexical properties relate to the concepts pertaining to the vocabulary used to name constituents. They characterise constituents with specific names, defined in a list of keywords. In future work, we plan to use the WORDNET lexical database of English to deal with synonyms.

Structural properties pertain to concepts related to the structure of the constituents of systems. For example, the property USE_GLOBAL_VARIABLE is used to check if a class uses global variables and the property NO_POLYMORPHISM to check if a class does not/prevents the use of polymorphism. System classes and interfaces characterised by the previous properties may be, in addition, linked with one another with three types of relationships: association, aggregation, and composition. Cardinalities define the minimum and the maximum numbers of instances of classes that participate in a relationship.

In the example of the Spaghetti Code, we obtain the following classification: measurable properties include the concepts of long methods, methods with no parameter, inheritance; lexical properties include the concepts of procedural names; structural properties include the concepts of global variables, polymorphism. Structural properties and relationships among constituents appear in the Blob and Functional Decomposition (see the key concepts depends on data and associated with small classes).

We also observe during the domain analysis that properties can be combined using set operators, such as intersection and union. For example, all properties must be present to characterise a class as Spaghetti Code.

\subsection{Classification of the key concepts}

Using the key concepts and their definitions, we build a taxonomy of smells by using all relevant key concepts to relate code and design smells on a single map and clearly identify their relationships.

We produce a map organising consistently smells and key concepts. This map is important to prevent misinterpretation by clarifying and classifying smells. It is similar in purpose to Gamma et al's Pattern Map [GHJV94, inside back cover]. For the sake of clarity, we show in Fig. 2 the taxonomy from the domain analysis of the four design smells shown in Table 1 only, which are used all through the paper. Figure 2 also includes the taxonomy of code smells associated with these design smells.

The taxonomy shows the structural relationships or set combinations (edges) among design smells (hexagons) and code smells (ovals in gray), and their relation with measurable, structural, and lexical properties (ovals in white). It gives an overview of all key concepts that characterise the four design smells and differentiates the key concepts as either structural relationships between code smells or their properties (measurable, structural, lexical). It also makes explicit the relationships among high- and low-level smells. Figure 3 gives the meta-model followed to design such a taxonomy. 


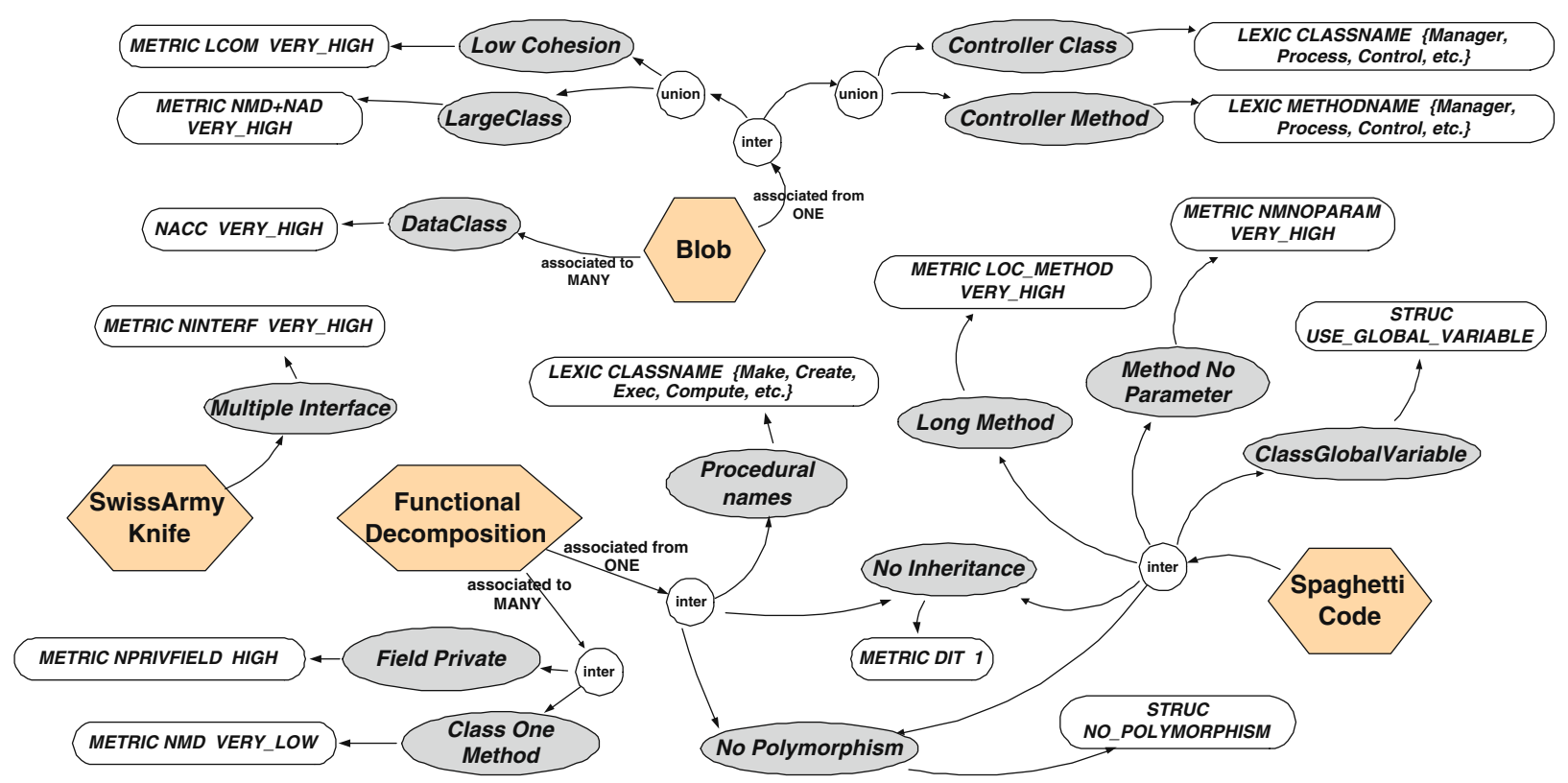

Figure 2. Taxonomy of smells. (Hexagons are design smells, gray ovals are code smells, white ovals are properties, and circles are set operators)

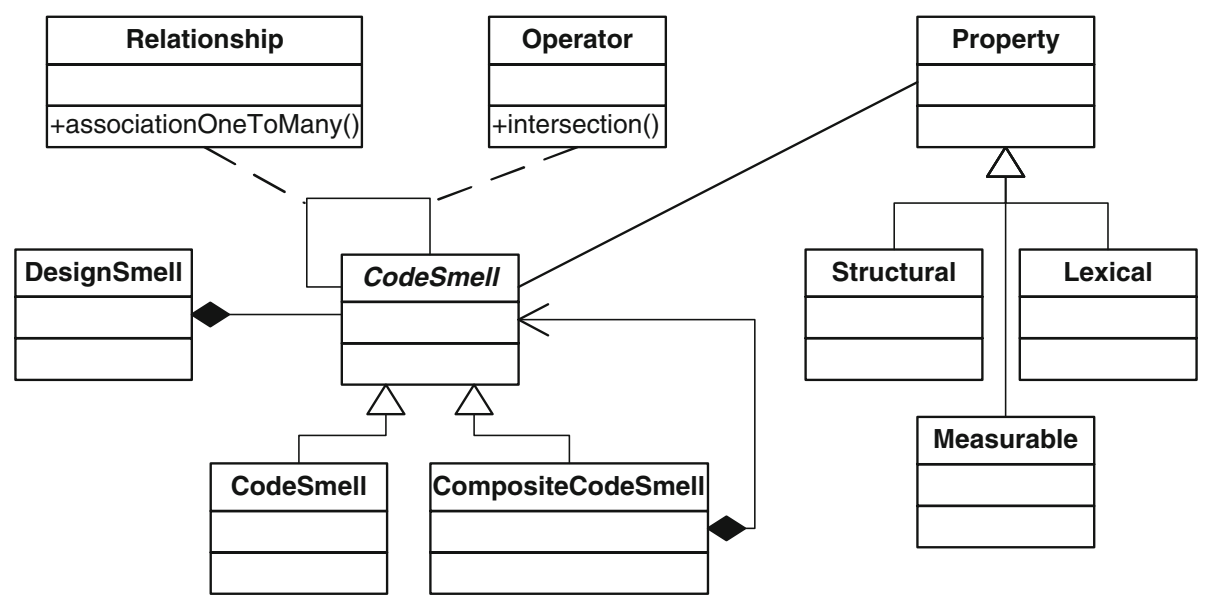

Figure 3. The taxonomy meta-model

\section{Specification of code and design smells}

The domain analysis performed in the previous section provides a set of key concepts, their definition, and a classification. Then, following Steps $2 \mathrm{a}$ and $2 \mathrm{~b}$ in Fig. 1, we design 2D-DSL, a domain-specific language (DSL) [MHS05] to specify smells in terms of their measurable, structural and lexical properties, and 2D- FW, a framework providing the services that are the operational implementations of the properties, set operations, and so on.

We build 2D-DSL and 2D- FW using and only using the identified key concepts, thus they both capture the domain expertise. Therefore, 2D-DSL and 2D- FW differ from general purpose languages and their runtime environments, which are designed to be universal [CM98]. They also differ from previous work on smell detection, which did not define explicitly a DSL and in which the detection framework drove the specification of the detection algorithms. 
From a domain analysis to the specification and detection of code and design smells

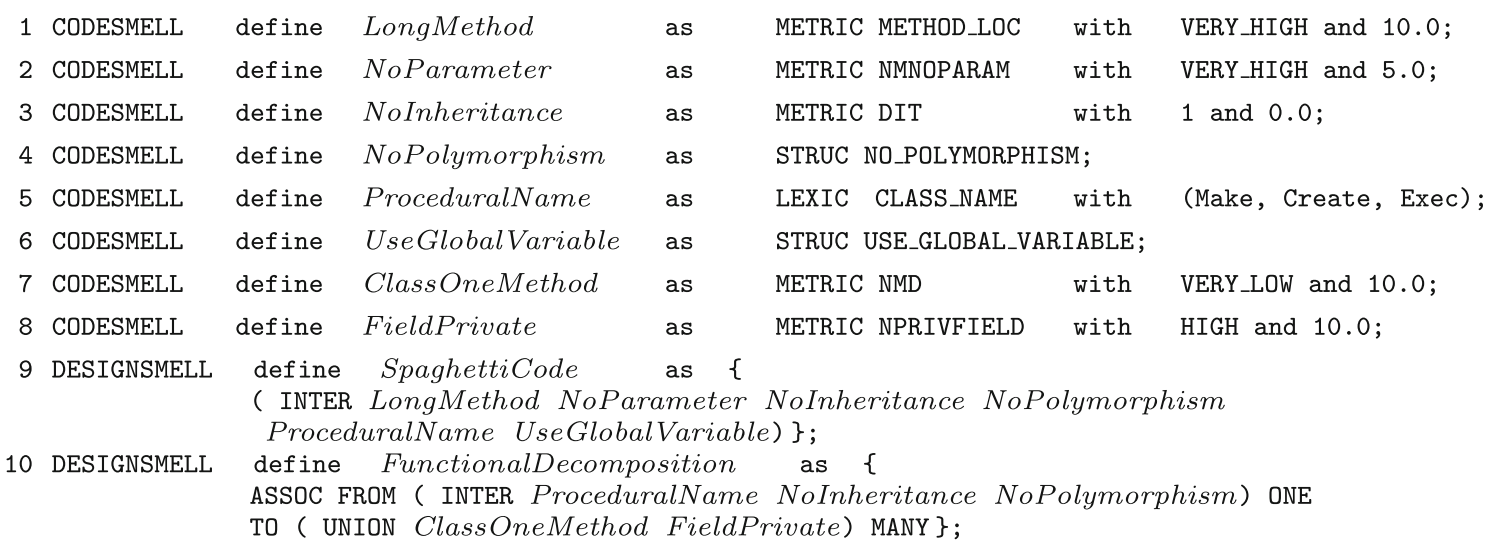

Figure 4. Specifications of the Spaghetti Code and Functional Decomposition.

Thus, with 2D-DSL and 2D- FW, it is easier for quality experts to understand smell specifications and to specify new smells because these are expressed using domain-related abstractions and focus on what to detect instead of how to detect it [CM98].

\subsection{D円SL}

With 2D-DSL, quality experts specify smells as sets of rules. Rules are expressed using key concepts. They can specify code smells, which correspond to measurable, lexical, or structural properties, or express design smells, which correspond to combinations of code smells using set operators or structural relationships. Figure 4 shows the specifications of the Spaghetti Code and Functional Decomposition design smells and their code smells. These two design smells shared some code smells as shown in the taxonomy in Fig. 2.

A Spaghetti Code is specified using the intersection of several rules (line 9). A class is Spaghetti Code if it declares methods with a very high number of lines of code (measurable property, line 1), with no parameter (measurable property, line 2); if it does not use inheritance (measurable property, line 3), and polymorphism (structural property, line 4), and has a name that recalls procedural names (lexical property, line 5), while declaring/using global variables (structural property, line 6). The float value after the keyword 'and' in measurable properties corresponds to the acceptable degree of fuzziness for this measure, which is the margin acceptable in percentage around the numerical value (line 3 ) or around the threshold relative to the ordinal value (lines 1-2). We further explain the ordinal values and the fuzziness in Sect. 4.

We formalise specifications with a Backus-Naur Form (BNF) grammar, shown in Fig. 5. A specification lists first a set of code smells and then a set of design smells (Fig. 5, line 1). A code smell is defined as a property. A property can be of three different kinds: measurable, structural, or lexical, and define pairs of identifier-value (lines 7-9). The BNF grammar specifies only a subset of possible structural properties, other can be added as new domain analyses are performed. The design smells are combinations of the code smells defined in the specification using set operators (lines 5-6). The design smells can also be linked to these code smells using relationships such as the composition, aggregation or association (lines 5, 21-23). Each code smell is identified by a name (line 2 ) and can be reused in the definition of design smells. Thus, the specification of the code smells, which can be viewed as a repository of code smells, can be reused or modified for the specification of different design smells.

\subsection{D-FW}

The 2D- FW framework provides the services that implement operations on the relationships, operators, properties, and ordinal values. It also provides services to build, access, and analyse systems. Thus, with 2D- FW, it is possible to compute metrics, analyse structural relationships, perform lexical and structural analyses on classes, and apply the rules. The set of services and the overall design of the framework are directed by the key concepts and the domain analysis. 2D- FW represents a super-set of previous detection frameworks and therefore could delegate part of its services to exiting frameworks. 


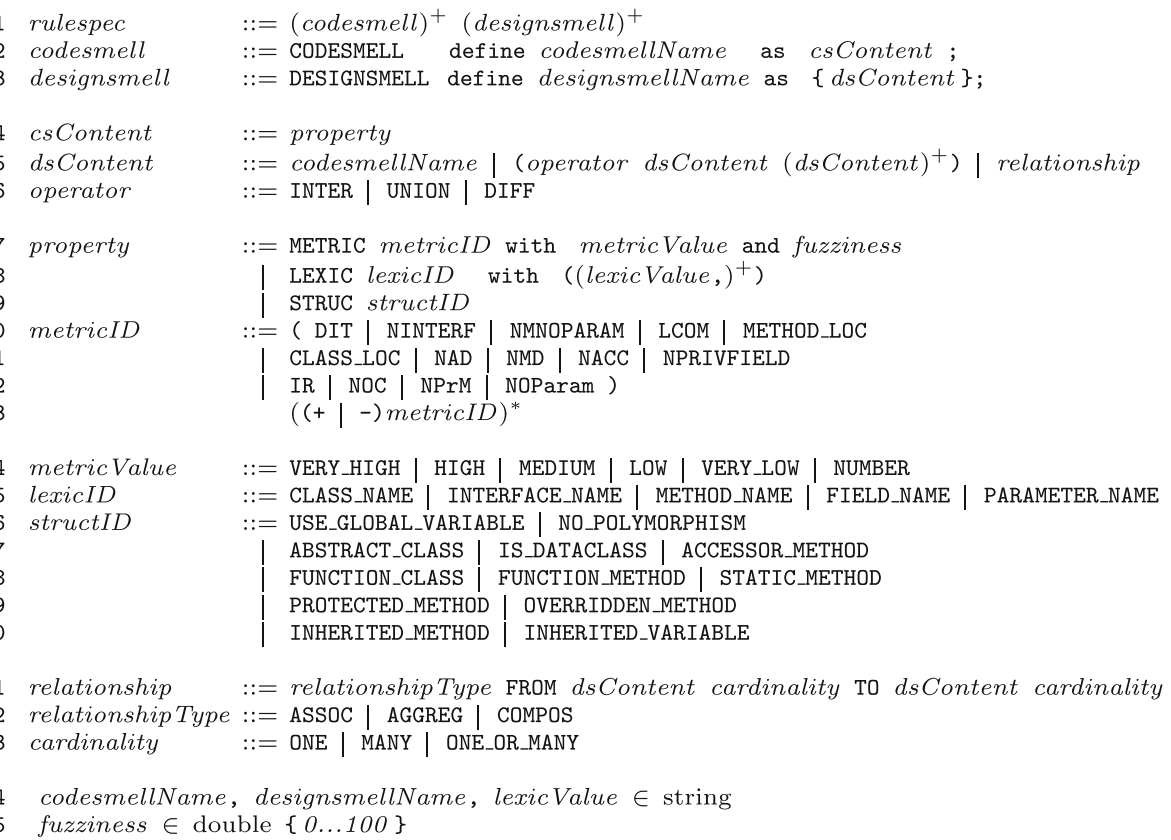

Figure 5. BNF grammar of smell specifications

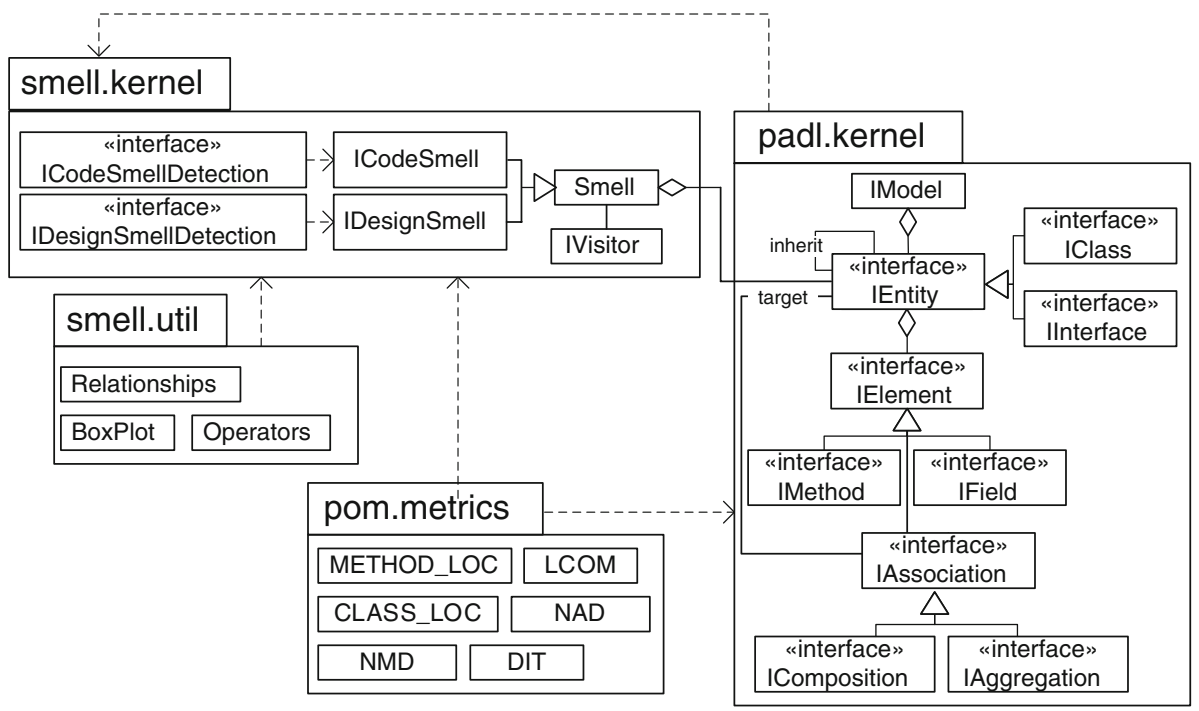

Figure 6. Architecture of the 2D- FW framework

2D- FW is built upon the PADL meta-model (Pattern and Abstract-level Description Language), a languageindependent meta-model to represent object-oriented systems, including binary class relationships [GAA04] and accessors, and on the POM framework (Primitives, Operators, Metrics) for metric computation [GSs04]. PADL offers a set of constituents from which we can build models of systems. It also offers methods to manipulate these models easily and generate other models, using the Visitor design pattern. We chose PADL because it is mature with 6 years of active development and is maintained in-house.

Figure 6 sketches the architecture of the 2D- FW framework. The architecture consists of two main packages, smell.kernel and smell.util. The smell.kernel package contains core classes and interfaces. Class Smell represents smells and is so far specialised in two subclasses, DesignSmell and CodeSmell. This hierarchy 
is consistent with our taxonomy of smells. A smell aggregates entities, interface IEntity from padl.kernel. For example, a smell is a set of classes with particular characteristics. Interfaces IDesignSmellDetection and ICodeSmellDetection define the services that detection algorithms must provide. The smell.util package declares utility classes that allow the manipulation of some key concepts of the specifications.

\subsection{Discussions}

The smell specifications are self-documenting and express naturally the textual descriptions of the smells. They can be modified by quality experts either to refine them by adding new rules or to modify existing ones to take into account the contexts of the systems, i.e., the set of information related to the characteristics of the analysed system. The context includes the type of systems (prototypes, systems in development or maintenance, industrial systems ...), design choices (related to principles or design heuristics), and coding standards (agreements to respect during the code writing). Quality experts can thus adapt the specifications to the context of their systems because the characteristics of systems differ from one system to the other, e.g., the quantity of code comments, which can be low in prototypes but high in system in maintenance, the permitted depth of inheritance, which differs according to the design choices, and the maximal size of classes and methods defined in coding standards. Thus, specifications can be modified easily at the domain level without any knowledge of the underlying detection framework.

In the cases where the domain-specific language would not provide the required constructs to specify a certain class of smells, it is possible to extend the language with a new set of primitives. However, in this case, the underlying detection framework should also be extended to provide the operational implementations of the new primitives. Such an extension can only be realised by developers modifying the detection framework using its general-purpose implementation language.

\section{From specifications to detection algorithms}

The problem that we solve is the automatic transition from the specifications to detection algorithms to avoid the manual implementation of algorithms, which is costly and not reusable, and to ensure the traceability between specifications and occurrences of detected smells. Thus, the automatic generation of detection algorithms spares the experts or developers of implementing by hand the detection algorithms and allows them to save time and resources.

The generation process consists of four fully automated steps, starting from the specifications through their reification to algorithm generation, as shown in Fig. 1, Steps 3a-3d, and detailed below.

\subsection{Parsing and reification}

The first step consists of parsing the smell specifications. A parser is built using JFLEX and JAVACUP ${ }^{2}$ from the BNF grammar, extended with appropriate semantic actions.

Then, as a specification is parsed, the second step consists of reifying the specifications based on the dedicated 2D- DL meta-model (Design Defects Definition Language). The meta-model is a representation of the abstract syntax tree generated by the parser. The meta-model 2D- DL defines constituents to represent specifications, rules, set operators, relationships among rules, and properties, as illustrated in Fig. 7. The result of this reification is a 2D- DL model of the specification, instance of class Specification defined in 2D- DL. An instance of Specification is composed of objects of type IRule, which describes rules that can be either simple or composite. A composite rule, CompositeRule, is a rule composed of other rules (Composite design pattern). Rules are combined using set operators defined in class Operator. Structural relationships are enforced using methods defined in class Relationship. The 2D- DL meta-model also implements the Visitor design pattern. This metamodel is quite similar to the meta-model of the taxonomy given in Fig. 3 and it illustrates the ease with which, from the taxonomy, we can specify intuitively the smells with the language.

\footnotetext{
${ }^{2}$ http://www2.cs.tum.edu/projects/cup/
} 


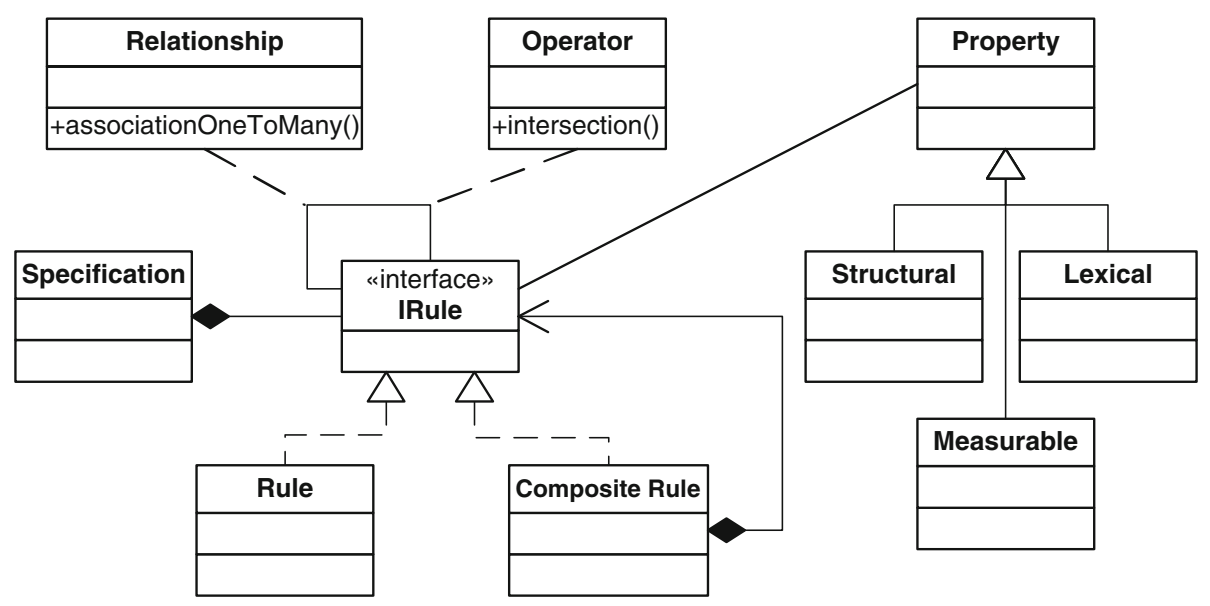

Figure 7. The 2D- DL meta-model

\subsection{Rule analyses}

This step consists of visiting the models of specifications and applying some consistency and domain-specific analyses. These analyses aim to identify incoherent or meaningless rules before generating the detection algorithms. Consistency analyses consist of verifying that specifications are not inconsistent, redundant, or incomplete. An inconsistent specification is, for example, two code smells defined with identical names but different properties. A redundant specification corresponds, for example, to two code smells defined with different names but identical properties. An example of an incomplete specification is a code smell referenced in the rule of an antipattern but not defined in the rule set of code smells. Domain-specific analyses consist of verifying that the rules are conform to the domain. For example, the value associated with a metric has a meaning in the domain: typically, a measurable property computed with the "metric number of methods declared" NMD compared to a float value has no meaning in the domain.

\subsection{Algorithm generation}

The generation of the detection algorithms is implemented as a set of visitors on models of specifications. The generation targets the services of the 2D- FW framework and is based on templates. Templates are excerpts of JAVA code source with well-defined tags to be replaced by concrete code. We use templates because our previous studies [MGL06, MHG06] showed that detection algorithms have recurring structures. Thus, we aggregate naturally all common structures of detection algorithms into templates. As we visit the model of a specification, we replace the tags with the data and values appropriate to the rules. The final source code generated for a specification is the detection algorithm of the corresponding smell and this code is directly compilable and executable without any manual interventions.

We detail in the following the generation of the detection algorithms of the set operators and the measurable properties. We do not present the generation of the lexical properties, structural properties, and structural relationships because they are similar to the ones presented here.

Measurable properties. The template given in Fig. 8e is a class called $\langle$ CODESMELL $>$ Detection that extends the class CodeSmellDetection and implements ICodeSmellDetection. It declares the method performDetection(), which consists of computing the specified metric on each class of the system. All the metric values are compared with one another with a boxplot, a statistical technique [CCKT83], to identify ordinal values, i.e., outlier or normal values. Then, the boxplot returns only the classes with metric values that verify the ordinal values. Figure $8 \mathrm{c}$ presents the process of generating code to verify a measurable property defined in the specification of the Spaghetti Code on a set of constituents. When the rule is visited in the model of the specification, we replace the tag <CODESMELL $>$ by the name of the rule, LongMethod, tag <METRIC > by the name of the metric, METHOD_LOC, 
From a domain analysis to the specification and detection of code and design smells

CODESMELL define LongMethod as

METRIC METHOD_LOC with VERY_HIGH and 10.0;
DESIGNSMELL define SpaghettiCode as \{ ((LongMethod $\cap$ NoParameter) ...

(a) Excerpt of the Spaghetti Code.

(b) Excerpt of the Spaghetti Code.

public void visit(IMetric aMetric) \{

replaceTAG ("<CODESMELL>", aRule.getName());

replaceTAG ("<METRIC>", aMetric.getName())

replaceTAG (<FUZZINESS〉,

aMetric.getFuzziness ());

replaceTAG (<ORDINAL_VALUE>,

\}

aMetric.getOrdinalValue())

private String getordinalValue(int value) \{

switch (value)

case VERY_HIGH

"getHighOutliers";

case HIGH :

"getHighValues" ;

case MEDIUM :

"getNormalValues";

(c) Visitor.

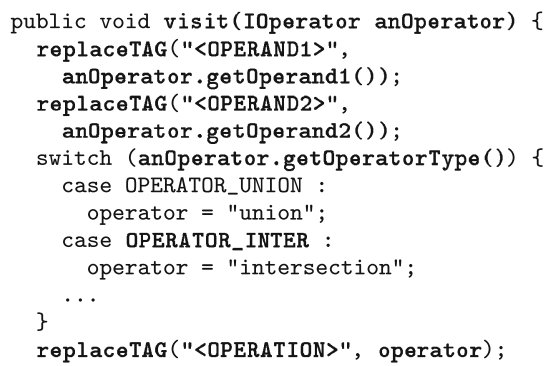

(d) Visitor.

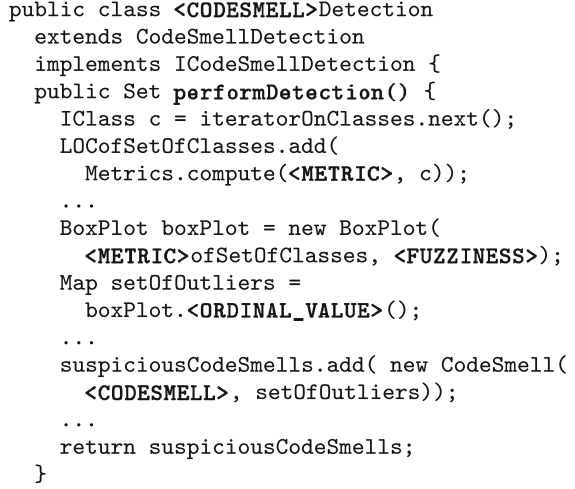

return suspiciousCodeSmells;

(e) Template.

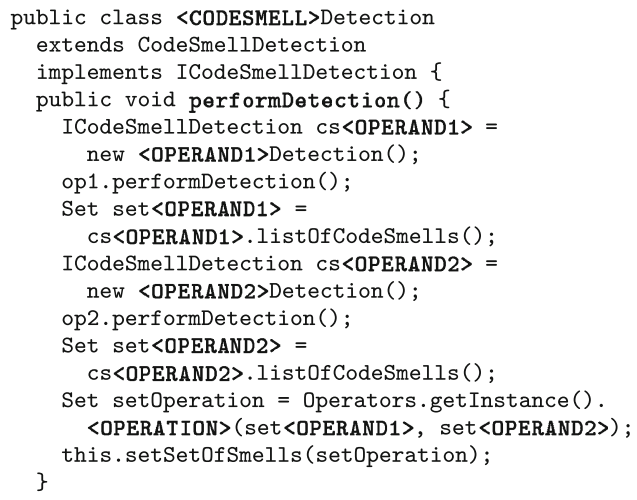

(f) Template.

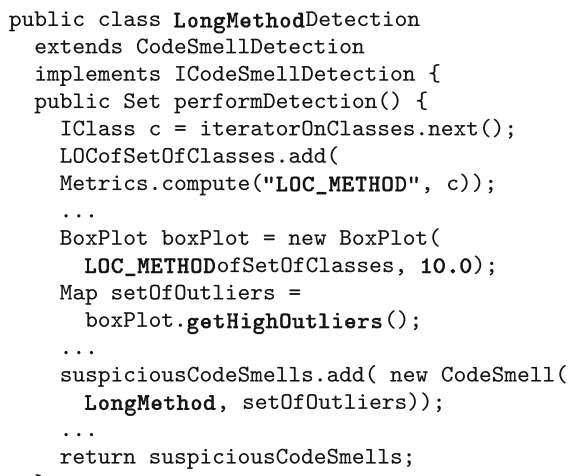

(g) Generated Code. public class Inter1

extends CodeSmellDetection implements ICodeSmellDetection \{ public void performDetection() \{

ICodeSmellDetection csLongMethod = new LongMethodDetection();

csLongMethod.performDetection();

Set setLongMethod $=$

csLongMethod.list0fCodeSmells();

ICodeSmellDetection csNoParameter =

new NoParameterDetection();

csNoParameter performDetection();

Set setNoParameter $=$

csNoParameter.listOfCodeSmells():

Set setOperation $=$ Operators.getInstance () .

intersection (setLongMethod, setNoParameter);

this.setSetOfSmells (setOperation):

(h) Generated Code.

Figure 8. Code generation for measurable properties (left) and set operators (right) 
tag <FUZZINESS > by the value 10.0, and tag <ORDINAL_VALUES > by the method associated with the ordinal value VERY_HIGH. Figure $8 \mathrm{~g}$ presents the code generated for the rule given in Fig. 8a.

Set operators. The rules can be combined using set operators such as the intersection in Fig. $8 \mathrm{~b}$. The code generation for set operators is quite different from the generation of properties. The template given in Fig.8f contains also a class called $<$ CODESMELL $>$ Detection that extends the class CodeSmellDetection and implements ICodeSmellDetection. However, the method performDetection() consists of combining with a set operator the list of classes in each operand of the rule given in Fig. 8b and returning classes that satisfy this combination. Figure $8 \mathrm{~d}$ presents the process related to the code generation for set operators in the specification of the Spaghetti Code. When an operator is visited in the model of the specification, we replace the tags associated with the operands of the rule, operand1: LongMethod, operand2: NoParameter, and the tag <OPERATION> by the type of set operator specified in the specification, i.e., intersection. The operands correspond to detection classes generated when visiting other rules as shown in Fig. 8h.

Discussion. The generated algorithms are by construct deterministic. We do not need to revise manually the code because the generation process ensures the correctness of the code source with respect to its specifications. This generated code tends sometimes itself towards Spaghetti Code and could be improved using polymorphism, yet it is automatically generated and is not intended to be read by quality experts and it will be improved in future work. We do not report the generation times because they take only few seconds.

\section{Validation}

We validate our contributions by specifying and detecting the ten smells presented in Sect. 2 and computing the precision and recall of the generated algorithms on GANTTPROJECT v1.10.2 and XERCES v2.7.0, two open-source systems. GANTTPROJECT v1.10.2 is a project-management tool to plan projects with Gantt charts. It contains 21,267 lines of code, 188 classes, and 41 interfaces. XERCES v2.7.0 is a framework for building XML parsers in Java. It contains 71,217 lines of code, 513 classes, and 162 interfaces.

We seek in the following to obtain a recall of $100 \%$ because quality experts need all smells to improve software quality and ease formal technical reviews. Having $100 \%$ recall means that all smells are detected and, thus, that quality experts can concentrate on the classes returned by the detection without needing to analyse by hand the classes not reported because, by definition, they cannot be part of a smell.

We are not aware of any other work reporting both precision and recall for smell detection algorithms.

\subsection{Validation process}

First, we build models of the analyzed systems. These models are obtained by reverse engineering. Then, we apply the generated detection algorithms on the models of the systems and obtain all suspicious classes that have potential smells. The list of suspicious classes is returned in a file. We validate the results of the detection algorithms by analysing the suspicious classes in the context of the complete model of the system and its environment. The validation is inherently a manual task. Therefore, we chose to apply the detection of the six code smells on GANTTPROJECT and of the four design smells on XERCES only so that we could divide the efforts of analysing manually these systems.

We recast the validation in the domain of information retrieval and use the measures of precision and recall, where precision assesses the number of true identified smells, while recall assesses the number of true smells missed by the algorithms [FBY92], according to the following equations:

$$
\begin{aligned}
& \text { precision }=\frac{\mid\{\text { existing smells }\} \cap\{\text { detected smells }\} \mid}{\mid\{\text { detected smells }\} \mid} \\
& \text { recall }=\frac{\mid\{\text { existing smells }\} \cap\{\text { detected smells }\} \mid}{\mid\{\text { existing smells }\} \mid}
\end{aligned}
$$

The computation of precision and recall is performed using independent results obtained manually because only quality experts can assess whether a suspicious class is indeed a smell or a false positive, depending on the specifications and the context and characteristics of the system. The manual analysis of GANTTPROJECT has been performed by one independent software engineer with a good expertise in code and design smells. We asked three 
From a domain analysis to the specification and detection of code and design smells

Table 3. Precision and recall of code smells in GantTPRoJeCt v1.10.2

\begin{tabular}{lccccc}
\hline Code smells & $\begin{array}{l}\text { Numbers of known } \\
\text { true positives }\end{array}$ & $\begin{array}{l}\text { Numbers of } \\
\text { detected smells }\end{array}$ & Precision (\%) & Recall (\%) & Time (s) \\
\hline Large class & $9(4.79 \%)$ & $13(6.91 \%)$ & 69.23 & 100.00 & 0.008 \\
Lazy class & $41(21.81 \%)$ & $104(55.32 \%)$ & 34.61 & 87.80 & 0.3 \\
Long method & $45(23.94 \%)$ & $22(11.70 \%)$ & 46.66 & 95.45 & 0.08 \\
Long parameter list & $54(28.72 \%)$ & $43(22.87 \%)$ & 79.63 & 100.00 & 0.030 \\
Refused parent bequest & $18(9.57 \%)$ & $20(10.64 \%)$ & 40.00 & 44.45 & 0.16 \\
Speculative Generality & $4(2.13 \%)$ & $4(2.13 \%)$ & 100.00 & 100.00 & 0.01 \\
\hline
\end{tabular}

In parenthesis, the percentage of classes affected by a smell. The number of classes in GANTTPROJECT v1.10.2 is 188 .

Table 4. Precision and recall of design smells in XERCES v2.7.0

\begin{tabular}{llllll}
\hline Design smells & $\begin{array}{l}\text { Numbers of known } \\
\text { True positives }\end{array}$ & $\begin{array}{l}\text { Numbers of } \\
\text { detected smells }\end{array}$ & Precision (\%) & Recall (\%) & Time (s) \\
\hline Blob & $39(7.60 \%)$ & $44(8.58 \%)$ & 88.64 & 100.00 & 2.45 \\
Functional decomposition & $15(2.92 \%)$ & $29(5.65 \%)$ & 51.72 & 100.00 & 0.91 \\
Spaghetti Code & $46(8.97 \%)$ & $76(14.81 \%)$ & 60.53 & 100.00 & 0.23 \\
Swiss Army Knife & $23(4.48 \%)$ & $56(10.91 \%)$ & 41.07 & 100.00 & 0.08 \\
\hline
\end{tabular}

In parenthesis, the percentage of classes affected by a smell. The number of classes in XERCES v2.7.0 is 513

master students and two independent software engineers to analyse manually XERCES using only Brown and Fowler's books to identify smells and compute the precision and recall of the algorithms. Each time a doubt on a candidate class arose, the software engineers considered the books as references in deciding by consensus whether or not this class was actually a smell. This task is tedious, some smells may have been missed by mistake, thus we have asked other software engineers to perform this same task to confirm our findings and on other systems to increase our database.

\subsection{Results}

Tables 4 and 3 report detection times, numbers of suspicious classes, and precisions and recalls, respectively for the GANTTPROJECT and XeRCES systems. We perform all computations on a Intel Dual Core at $1.67 \mathrm{GHz}$ with $1 \mathrm{~Gb}$ of RAM. Computation times do not include building the models of the system but include accesses to compute metrics and check structural relationships and lexical and structural properties.

Excluding the code smells Lazy Class and Refused Parent Bequest, the recalls of the generated algorithms range from 95.45 to $100 \%$ for code smells and $100 \%$ for design smells. Precisions range from $46.66 \%$ to $100 \%$, providing between 2.13 and $22.87 \%$ of the total number of classes, which is reasonable for quality experts to analyse by hand, with respect to analysing the entire system, 188 classes GANTTPROJECT, manually.

For the Spaghetti Code, we found 76 suspicious classes. Out of these 76 suspicious classes, 46 are indeed Spaghetti Code previously identified in XERCES manually by software engineers independent of the authors, which leads to a precision of $60.53 \%$ and a recall of $100.00 \%$ (see third line in Table 4). The result file contains all suspicious classes, including class org.apache.xerces.xinclude.XIncludeHandler declaring 112 methods. Among these 112 methods, method handleIncludeElement (XMLAttributes) is typical of Spaghetti Code: it does not use inheritance and polymorphism, uses excessively global variables, and weighs 759 LOC.

For the Large Class, Long Parameter List, and Speculative Generality code smells, we obtained optimum recalls because the generated detection algorithms are based on quite simple metrics that consist in counting entities. For the Long Method, the same kind of simple metric, the number of lines of code, has been used. However, this metric depends on the experts' definition of a long method, which explains that we did not obtain a perfect recall $(95.45 \%)$.

We encountered another ambiguity with Lazy Class. Indeed, one of the criteria of its detection is method complexity: during manual detection, software engineers must appreciate method complexity, which is inherently subjective, while we use objective metrics (WMC, Weighted methods per class, and McCabe Cyclomatic Complexity) during the automatic detection.

Finally, the smell Refused Parent Bequest illustrated the inverse problem: it is very difficult for software engineers to identify all its occurrences because they must appreciate if a class uses enough public and protected methods/fields of any of its superclasses. Moreover, the software engineers consider bequests provided by API classes whereas we apply our detection on the system only, excluding libraries. 
The two smells Lazy Class and Refused Parent Bequest exhibit poorer precision and recall because of the lack of constraints of their specifications. A Lazy Class could be any class with few simple methods while a Refused Parent Bequest could be any class that does not directly use some of its parents methods. These two smells illustrate the problems of interpreting the definitions of the smells and of translating these definitions into specifications. Future work includes studying the contexts in which these specifications could be made more constraining.

The results depend on the specifications of the smells. The specifications must be neither too loose, not to detect too many suspicious classes, nor too constraining, and miss smells. With 2D-DSL, quality experts can refine the specifications of the smells easily, according to the detected suspicious classes and their knowledge of the system through iterative refinement.

\subsection{Discussions}

Results. The validation shows that the specifications of the design and code smells lead to generated detection algorithms with expected recalls and good precisions. Thus, it confirms that: (1) the language allows describing several smells. We described ten smells including 4 different design smells and six code smells; (2) the generated detection algorithms have, mainly, a recall of $100 \%$, i.e., all known smells are detected, and an average precision greater than $34 \%$, i.e., the detection algorithms report less than $2 / 3$ of false positives with respect to the number of true positives. Quality experts do not need to look at classes not detected as smells because they cannot be part of smells thanks to the recall of $100 \%$; and, (3) the complexity of the generated algorithms is reasonable, i.e., the generated algorithms have computation times of few seconds. Computation is fast because the complexity of our detection algorithms depends only on the number of classes in the system, $n$, and on the number of properties to verify: the complexity of the generated detection algorithms is $(c+o p) \times \mathcal{O}(n)$, where $c$ is the number of properties and $o p$ of operators.

Threats to the validity. The validity of the results depends directly on the smell specifications. We performed our experiments on a representative set of smells to lessen the threat to the internal validity of our validation. The subjective nature of interpreting, specifying, and identifying smells is a threat to the construct validity of this validation. We lessen this threat by specifying the smells based on the literature and by involving several independent software engineers in the computation and manual assessment of the results. The threat to external validity is related to the exclusive use of open-source JAVA systems, which may prevent the generalisation of our results to other systems. Nevertheless, we chose to perform our experiments on freely available systems to allow their verification and replication. Furthermore, in the context of a cooperation with a company (not described here for confidentiality reasons), we also applied our approach on their proprietary systems and we obtained similar encouraging results.

\section{Related work and current state-of-the-art}

Several smell detection approaches and tools have been proposed in the literature to specify and detect code and design smells. However, we are not aware of any approach that is based on an explicit domain analysis and its resulting domain-specific language. Moreover, most of these approaches do not clarify the process that allows the detection of smells from their specifications. These approaches are often driven by the services of their underlying detection framework rather than by an exhaustive study of the descriptions of smells that led to their specifications. Finally, if others explain their detection process, they do not provide a validation of their detection technique. We first present the related work and then a detailed comparison of our approach with the approach of detection strategies [Mar04], the current state-of-the-art.

\subsection{Related work}

Several books provide in-breadth views on pitfalls [Web95], heuristics [Rie96], code smells [Fow99], and antipatterns $\left[\mathrm{BMB}^{+} 98\right]$ aimed at a wide audience for educational purposes. However, they describe textually smells but none performed a complete analysis of the text-based descriptions of smells. Thus, it is difficult to build detection algorithms from their textual descriptions because they lack precision and are prone to misinterpretation. Travassos et al. [TSFB99] introduced a process based on manual inspections and reading techniques to identify 
From a domain analysis to the specification and detection of code and design smells

Table 5. Precision and recall of code smells in GANTTPRoJECT v1.10.2 using iPlasma

\begin{tabular}{lclll}
\hline Code smells & $\begin{array}{l}\text { Numbers of known } \\
\text { True positives }\end{array}$ & $\begin{array}{l}\text { Numbers of } \\
\text { detected smells }\end{array}$ & Precision (\%) & Recall (\%) \\
\hline Large class & $9(4.79 \%)$ & $1(0.53 \%)$ & 100.00 & 11.11 \\
Lazy class & $41(21.81 \%)$ & $\varnothing$ & $\varnothing$ & $\varnothing$ \\
Long method & $45(23.94 \%)$ & $11(5.85 \%)$ & 100.00 & 24.44 \\
Long parameter list & $54(28.72 \%)$ & $44(23.40 \%)$ & 100.00 & 81.48 \\
Refused parent bequest & $18(9.57 \%)$ & $1(0.53 \%)$ & 0 & 0 \\
Speculative generality & $4(2.13 \%)$ & $9(4.79 \%)$ & 100.00 & 44.44 \\
\hline
\end{tabular}

smells. No attempt was made to automate this process and thus it does not scale to large systems easily. Also, it only covers the manual detection of smells, not their specification.

Marinescu introduced the concept of detection strategies to overcome the limitations of the previous literature. We present a detailed comparison of our approach with these detection strategies in the following subsection. Munro [Mun05] also noticed the limitations of the textual descriptions and proposed a template including heuristics to describe code smells more systematically. It is a step towards more precise specifications of code smells but code smells remain nonetheless textual descriptions subject to misinterpretation. Munro also proposed metric-based heuristics to detect code smells, which are similar to Marinescu's detection strategies. Only Marinescu [Mar04] and Munro [Mun05] provide some results of their detection but on a few smells and only on proprietary systems. Alikacem and Sahraoui proposed a description language of quality rules to detect violations of quality principles and smells [AS06]. The rules include quantitative information such as metrics and structural information such as inheritance or association relationships among classes. They also use fuzzy logic to express the thresholds of rules conditions. The rules are interpreted and executed using an inference engine. Although their detection framework is explicit, they did not provide any validation of their approach. All these approaches have contributed significantly to the automatic detection of smells. However, none presents a complete solution including a specification language, explicit detection framework detailed processing, and validation of the detection technique.

Tools such as PMD [PMD02], CHECKSTYLE [Che04], and FXCOP [FXC06] detect problems related to coding standards, bugs patterns or unused code. Thus, they focus on implementation problems but do not address higherlevel design smells such as antipatterns. PMD [PMD02], SEMMLECode [Sem07] and HaMmURAPI [Ham07] also allow developers to write detection rules using JAVA or XPATH. However, the addition of new rules is intended for engineers familiar with JAVA and XPATH, which could limit access to a wider range of software engineers. CROCOPAT [BNL05] provides an efficient language to manipulate relations of any arity with a simple and expressive query and manipulation language. However, this language is at a low-level of abstraction and requires quality experts to understand the implementation details of its underlying model. Therefore, previous tools provided low-level languages for specifying new smells while we defined a high-level DSL targeting software quality experts.

\subsection{Comparison with IPlasma}

Marinescu [Mar04] presented a metric-based approach to detect code smells with the concept of detection strategies and implemented a tool called IPLASMA. Detection strategies capture deviations from good design principles. They are described with a medium-level specification language based on metrics through a simple process. They are therefore a step towards the precise specifications of code smells and, as such, have been often cited. Therefore, we considered as others detection strategies and IPLASMA the current state-of-the-art. Yet, detection strategies have limitations. First, the specifications are mainly metric-based and, thus for example, cannot describe specific relationships between classes of interest. Second, the specifications are hard-coded in the tool and are not readily available for study and - or modification.

To further compare the two approaches, we have proceeded to the detection of the six code smells presented in Table 2 using IPLASMA on GantTPROJECT as for our approach in Table 3. Table 5 presents the results of this detection.

First, we notice that IPLASMA also uses the technique of the boxplot to evaluate relative values specified in the detection strategies. However, the mapping from the relative values of the boxplot with the metric values is not explicit. Therefore, we could not detect occurrences of Lazy Class because it was not possible to define explicitly 
rules using the bloxplot. In our approach, we make explicit the boxplot and enhance it with fuzzy logic and, thus, alleviates the problem related to the definition of thresholds.

Second, IPLASMA focuses on a high precision rather that a high recall. This is an appropriate choice in the context of a day-to-day use by developers as part of their development process. In our approach, we focus on a high recall but developers could adapt the detection algorithms to enforce a high precision using our high-level domain-specific language.

Third, the results of the detection of occurrences of Refused Bequest Parent in terms of precision and recall are surprising. IPLASMA only detects one (false-positive) occurrence while, our approach provides a $40 \%$ precision and $44 \%$ recall. We cannot explain this poor performance of IPLASMA for lack of access to the specifications of the smells. Other code smells exhibit perfect precisions and recalls between 11 and $81 \%$.

Finally, although the computation times in IPLASMA are similar to those of our approach (typically less than a second), IPLASMA requires user interactions to select the smells and the programs in which to detect their occurrences while our approach can be fully automated and integrated in the developers' development environment.

In conclusion, we drew much inspiration from the concept of detection strategies and from their implementation in IPLASMA to design a high-level domain specific language and its companion generation algorithms. We thus attempted to overcome the limitations of this approach illustrated in this comparison.

\section{Conclusion and future work}

In this paper, we introduced a domain analysis of the key concepts defining design and code smells; a domainspecific language to specify smells, 2D-DSL, and its underlying detection framework, 2D- FW; and a process for generating detection algorithms automatically.

With 2D-DSL, 2D- FW, and the generation process, quality experts can specify smells at the domain level using their expertise, generate detection algorithms, and detect smells during formal technical reviews. We implemented 2D-DSL, 2D- FW, and the generation process and studied the precision and recall of 10 generated algorithms on GANTTPROJECT v1.10.2 and XERCES v2.7.0. We showed that the detection algorithms are efficient and precise, and have a recall close to $100 \%$ recall.

The validation in terms of precision and recall sets a landmark for future quantitative comparisons. Thus, we plan to perform such a comparison of our work with previous approaches in complement to the comparison with IPLASMA described here. We also plan to integrate the WORDNET lexical database of English into 2D- FW, to improve the current DSL, to improve the generated code in terms of quality and reusability, and to compute the precision and recall on more systems. We will assess the flexibility of the code generation offered when using $\mathrm{XML}$ technologies $\left[\mathrm{SPJ}^{+} 05\right]$. We will also perform usability studies of the language with quality experts.

\section{Acknowledgements}

We thank Giuliano Antoniol, Kim Mens, Dave Thomas, and Stéphane Vaucher for fruitful discussions. We also thank the master students and software engineers who performed the manual analysis and Duc-Loc Huynh and Pierre Leduc for their help in building 2D- FW.

\section{References}

[AS06] Alikacem El H, Sahraoui H (2006) Détection d'anomalies utilisant un langage de description de règle de qualité. In: actes du $12^{e}$ colloque LMO, pp 185-200

$\left[\mathrm{BMB}^{+} 98\right]$ Brown WJ, Malveau RC, Brown WH, McCormick III HW, Mowbray TJ (1998) Anti patterns: refactoring software, architectures, and projects in crisis, 1st edn. Wiley, New York

[BNL05] Beyer D, Noack A, Lewerentz C (2005) Efficient relational calculation for software analysis. Trans Softw Eng 31(2):137-149

[CCKT83] Chambers JM, Clevelmd WS, Kleiner B, Tukey PA (1983) Graphical methods for data analysis. Wadsworth International

[Che04] CheckStyle (2004) http://checkstyle.sourceforge.net

[CK94] Chidamber SR, Kemerer CF (1994) A metrics suite for object oriented design. IEEE Trans Softw Eng 20(6):476-493

[CM98] Consel C, Marlet R (1998) Architecturing software using: A methodology for language development. Lecture Notes in Computer Science, vol 1490, pp 170-194

[FBY92] Frakes WB, Baeza-Yates R (1992) Information retrieval: data structures and algorithms. Prentice-Hall, Englewood Cliffs

[Flo02] Florijn G (2002) Revjava-design critiques and architectural conformance checking for java software. White Paper. SERC, the Netherlands 
From a domain analysis to the specification and detection of code and design smells

[Fow99] Fowler M (1999) Refactoring - improving the design of existing code, 1st edn. Addison-Wesley, Reading

[FXC06] FXCop (2006) http://www.gotdotnet.com/team/fxcop/

[GAA04] Guéhéneuc Y-G, Albin-Amiot H (2004) Recovering binary class relationships: Putting icing on the UML cake. In: Proceedings of the 19th OOSPLA conference, pp 301-314

[Ger91] Geraci A (1991) IEEE standard computer dictionary: compilation of IEEE standard computer glossaries. Institute of Electrical and Electronics Engineers, Inc.

[GHJV94] Gamma E, Helm R, Johnson R, Vlissides J (1994) Design patterns —elements of reusable object-oriented software, 1st edn. Addison-Wesley, Reading

[GSs04] Guéhéneuc Y-G, Sahraoui H, Zaidi F (2004) Fingerprinting design patterns. In: Proceedings of the 11th WCRE conference, pp 172-181

[Ham07] Hammurapi, October 2007. http://www.hammurapi.biz/

[HP04] Hovemeyer D, Pugh W (2004) Finding bugs is easy. SIGPLAN Not. 39(12):92-106

[LLL08] Lincke R, Lundberg J, Löwe W (2008) Comparing software metrics tools. In: Proceedings of the ISSTA Symposium, NY, USA, 2008. ACM, New York, pp 131-142

[Mar04] Marinescu R (2004) Detection strategies: metrics-based rules for detecting design flaws. In: Proceedings of the 20th ICSM conference, pp 350-359

[MGL06] Moha N, Guéhéneuc Y-G, Leduc P (2006) Automatic generation of detection algorithms for design defects. In: Proceedings of the 21st ASE conference

[MGMD08] Moha N, Guéhéneuc Y-G, Le Meur A-F, Duchien L (2008) A domain analysis to specify design defects and generate detection algorithms. In: Proceedings of the 11th FASE conference. Springer, Heidelberg, pp 276-291

[MHG06] Moha N, Huynh D-L, Guéhéneuc Y-G (2006) Une taxonomie et un métamodèle pour la détection des défauts de conception. In: actes du $12^{e}$ colloque LMO, pp 201-216

[MHS05] Mernik M, Heering J, Sloane AM (2005) When and how to develop domain-specific languages. ACM Comput Surv 37(4): 316-344

[Mun05] Munro MJ (2005) Product metrics for automatic identification of "bad smell" design problems in java source-code. In: Proceedings of the 11 th metrics symposium

[PD90] Prieto-Díaz R (1990) Domain analysis: an introduction. Softw Eng Notes 15(2):47-54

[PMD02] PMD (2002) http://pmd.sourceforge.net/

[Pre01] Pressman RS (2001) Software engineering — a practitioner's approach. McGraw-Hill Higher Education, 5th edn. November 2001

[Rie96] Riel AJ (1996) Object-oriented design heuristics. Addison Wesley, Reading

[Sem07] SemmleCode, October 2007. http://semmle.com/

[SPJ ${ }^{+}$05] Swint GS, Pu C, Jung G, Yan W, Koh Y, Wu Q, Consel C, Sahai A, Moriyama K (2005) Clearwater: extensible, flexible, modular code generation. In: Proceedings of the 20th IEEE/ACM ASE conference, pp 144-153

[TSFB99] Travassos G, Shull F, Fredericks M, Basili VR (1999) Detecting defects in object-oriented designs: using reading techniques to increase software quality. In: Proceedings of the 14th OOSPLA conference, pp 47-56

[WBM02] Wirfs-Brock R, McKean A (2002) Object design: roles, responsibilities and collaborations. Addison-Wesley, Reading

[Web95] Webster BF (1995) Pitfalls of object oriented development. M\&T Books, New York

Received 10 October 2008

Accepted in revised form 21 April 2009 by J.L. Fiadeiro, P. Inverardi and T.S. E. Maibaum 